\title{
EFEITO DE VISITAS DOMICILIARES NA \\ MANUTENÇÃO DAS ATIVIDADES DE VIDA \\ DIÁRIA DE ADULTOS EM PÓS-OPERATÓRIO
}

\author{
EFFECT OF HOME VISITS IN THE \\ MAINTENANCE OF DAILY LIFE ACTIVITIES \\ OF ADULTS IN POSTOPERATIVE PERIOD
}

\section{EFECTO DE LAS VISITAS DOMICILIARIAS EN EL MANTENIMIENTO DE LAS ACTIVIDADES DE LA VIDA DIARIA DE LOS ADULTOS EN EL POSTOPERATORIO}

\author{
António Manuel Rocha Fonseca Pinto ${ }^{1}$ \\ Elisabete Pimenta Araújo Paz ${ }^{2}$ \\ Amâncio António de Sousa Carvalho ${ }^{3}$
}

Como citar este artigo: Pinto AMRF, Paz EPA, Carvalho AAS. Efeito de visitas domiciliares na manutenção das atividades de vida diária de adultos em pós-operatório. Rev baiana enferm. 2019;33:e33413.

Objetivo: avaliar os efeitos da implantação de um programa de visitas domiciliares (VD) a pessoas adultas no domínio Atividades de Vida Diária (AVD). Método: estudo quasi-experimental, com usuários de um centro hospitalar do Norte de Portugal, aos quais se aplicou a escala Hip Disability and Osteoarthritis Outcome Score LK2.0. O tratamento de dados foi realizado pelo SPSS. Resultados: a maioria dos participantes era do sexo masculino (61,4\%) e pertencia à faixa etária dos 65 anos ou mais (65,9\%). Não se verificou um efeito significativo na interação do programa de VD, sobre o compósito multivariado (AVD Observação1 e AVD Observação2) MANOVA: p=0,164, mas a média da pontuação do domínio AVD nos dois grupos, em comparação, aumentou da Observação1 para a Observação2, sendo o aumento mais pronunciado no Grupo Experimental. Conclusão: o programa de VD não teve efeito significativo na melhora do domínio AVD, mas contribuiu ligeiramente para a autonomia do usuário.

Descritores: Osteoartrite do Quadril. Atividades Cotidianas. Intervenção Precoce (Educação). Enfermagem em Saúde Comunitária. Visita Domiciliar.

Objective: to evaluate the effects of implementing a home visiting (HV) program for adults in the Daily Life Activities (DLA) domain. Method: quasi-experimental study with users of a hospital center in north Portugal, with the application of the Hip Disability and Osteoarthritis Outcome Score LK2.O scale. Data processing was performed through SPSS. Results: most participants were male (61.4\%) and were 65 years old or older (65.9\%). There was no significant effect on HV program interaction, on multivariate composite (DLA Observation1 and DLA Observation2) MANOVA: $p=0.164$, but average DLA domain score in both groups increased from Observation 1 to Observation2, with the most

Enfermeiro. Mestre em Enfermagem Comunitária. Centro Hospitalar de Trás-os-Montes e Alto Douro. Vila Real, Portugal. https://orcid.org/0000-0002-I272-I679 Enfermeira. Doutora em Enfermagem. Professora da Universidade Federal do Rio de Janeiro. Rio de Janeiro, Rio de Janeiro, Brasil. bete.paz@gmail.com. https:// orcid.org/0000-0002-1692-0253

3 Enfermeiro. Doutor em Estudos da Criança. Professor da Universidade de Trás-os-Montes e Alto Douro. Vila Real, Portugal. amanciocarv@hotmail.com http://orcid. org/0000-0002-1573-5312 
pronounced increase in the Experimental Group. Conclusion: the HV program had no significant effect on improving the DLA domain, but slightly contributed to the user's autonomy.

Descriptors: Osteoarthritis, Hip. Activities of Daily Living. Early Intervention (Education). Community Health Nursing. House Calls.

Objetivo: evaluar los efectos de implementar un programa de visitas domiciliarias (VD) para adultos en el dominio de actividades de la vida diaria (AVD). Método: estudio cuasiexperimental con usuarios de un hospital en el norte de Portugal, con la aplicación de la escala Hip Disability and Osteoarthritis Outcome Score LK2.O. El procesamiento de datos se realizó a través de SPSS. Resultados: la mayoría de los participantes eran hombres $(61,4 \%)$ y tenían 65 años o más (65,9\%). No bubo un efecto significativo sobre la interacción del programa VD en el compuesto multivariado (AVD Observation1 y AVD Observation2) MANOVA: $p=0.164$, pero el puntaje promedio del dominio AVD en ambos grupos aumentó de Observation1 a Observation2, siendo el aumento más pronunciado en el Grupo Experimental. Conclusión: el programa de VD no tuvo un efecto significativo en la mejora del dominio AVD, pero contribuyó ligeramente a la autonomía del usuario.

Descriptores: Osteoartritis de la Cadera. Actividades Cotidianas. Intervención Temprana (Educación). Enfermería en Salud Comunitaria. Visita Domiciliaria.

\section{Introdução}

Nas últimas décadas, o envelhecimento da população transformou-se em um fenômeno de amplitude mundial. No plano demográfico, tornou-se um dos principais fatores da alteração da estrutura da população, isto é, da inversão da pirâmide demográfica. Até ao ano de 2050, uma em cada cinco pessoas terá 60 anos ou mais, totalizando dois bilhões de pessoas em todo o mundo ${ }^{(1)}$. Em 2015, os idosos já representavam $12 \%$ da população mundial, com previsão de duplicar esse quantitativo até $2050^{(2)}$.

Uma das consequências da elevação dos padrões de aumento da longevidade é o aumento da carga de doenças de natureza crônico-degenerativa. Estas, por sua vez, estão intimamente relacionadas ao aumento de sequelas que provocam algum tipo de limitação na realização de Atividades da Vida Diária (AVD). A Osteoartrite (OA) é a mais importante doença reumática, afetando todos os componentes das articulações. É uma das dez doenças mais incapacitantes em países desenvolvidos, sendo uma das principais causas de dor e incapacitação em todo o mundo. E, em âmbito mundial, estima-se que 9,6\% dos homens e 18\% das mulheres com idade acima de 60 anos padecem de OA sintomática ${ }^{(3)}$.

Outros autores também afirmam que a OA é a doença articular mais comum no mundo e sua prevalência aumenta com a idade ${ }^{(4-5)}$. Estima-se que, no Brasil, afete cerca de $6 \%$ a $12 \%$ dos adultos e mais de um terço dos idosos, sendo considerada uma doença que gera altos custos para os sistemas de saúde ${ }^{(4)}$. Segundo a Liga Portuguesa Contra as Doenças Reumáticas, nos países industrializados, as doenças reumáticas e músculo-esqueléticas afetam mais pessoas do que qualquer outro grupo de doenças. Atinge um quarto de todas as pessoas na União Europeia, perfazendo mais de 120 milhões de pessoas, sendo a maior causa de faltas laborais por doença e de aposentadoria antecipada em todo o mundo. Nessa região, a despesa pública com essas doenças totaliza mais de 200.000 milhões de euros por ano, o que as tornam as doenças mais caras para os sistemas socioeconômicos e de saúde europeus ${ }^{(6)}$.

Dados de nove estudos portugueses realizados entre 1976 e 2000 indicam que as doenças de origem reumática são a patologia crônica mais prevalente, representando 28\% a 37\% das patologias crônicas na população portuguesa. Estes resultados estão em consonância com outros estudos realizados em Portugal no mesmo período, em que as queixas relacionadas com o sistema músculo-esquelético corresponderam a 29,5\% dos principais problemas de 
saúde. A osteoartrose corresponde a 3,8\% dos motivos de consulta em unidades de Cuidados de Saúde Primários, sendo a segunda causa de gastos totais em medicamentos, a primeira causa de afastamentos laborais e do total de dias de licenças por doença, a primeira causa de incapacidade temporária/incapacidade definitiva e, em última análise, a primeira causa de aposentadorias ${ }^{(7)}$.

A OA é uma doença articular crônico-degenerativa que se evidencia pelo desgaste da cartilagem articular, caraterizando-se por dor, principalmente no período da manhã, rigidez matinal, crepitação óssea e atrofia muscular, tendo como fatores de risco idade, sexo feminino, obesidade, sobrecarga articular excessiva, trauma, lesões periarticulares, alguns riscos ocupacionais e componentes genéticos ${ }^{(5)}$.

A dor surge inicialmente com o movimento da articulação, seguindo-se um aumento da sua frequência e intensidade. Posteriormente, é sentida pelo paciente, ainda que em repouso. Com o agravamento da doença, surgem rigidez articular, deformidade, sinais inflamatórios, crepitação articular, limitação do movimento articular e claudicação na marcha, sinal com tendência para se agravar com o decorrer do tempo, aumentando a incapacidade funcional na realização das AVD e, como consequência, diminuindo a qualidade de vida. A coxartrose está na origem da maioria das Artroplastias Totais do Quadril (ATQ) realizadas anualmente nos EUA e em Portugal ${ }^{(8)}$.

Após a alta hospitalar, o usuário submetido a ATQ ainda mostra limitações na realização das suas AVD. A visita domiciliar (VD), por parte da enfermagem, é uma atividade necessária e de grande importância no auxílio para o regresso a uma vida "normal", visando a promoção da saúde, numa transição saúde-doença e doença-saúde ${ }^{(9)}$, mediante um cuidado integral, resolutivo e contextualizado, adequado à cultura e à dinâmica familiar do usuário ${ }^{(10)}$. A VD em Portugal pode ser de caráter multidisciplinar ou de cada um dos profissionais de saúde, mas são os Enfermeiros que mais realizam essa atividade.

A VD de enfermagem é, pois, um instrumento importante e muito utilizado no processo de Educação em Saúde (ES), no qual se estabelece um conjunto de saberes e práticas de enfermagem orientadas para a prevenção da doença e promoção da saúde, constituindo uma estratégia no cotidiano de trabalho, que pode desencadear mudança de atitudes e de comportamentos, possibilitando a melhoria da qualidade de vida e a autonomia dos usuários envolvidos. É, por isso, complexo em sua exequibilidade, sendo essencial para devolver autonomia nos cuidados e consequente melhora na qualidade de vida das pessoas ${ }^{(11)}$.

A enfermagem exerce, na atualidade, um papel extremamente importante na satisfação das necessidades dos pacientes, particularmente as de informação e formação, após esses terem sido submetidos a ATQ. O corpo de conhecimentos científicos que o enfermeiro detém permite-lhe desenvolver competências que lhe possibilitam, por meio da VD, orientar, instruir, treinar e fornecer os meios para que o indivíduo, a família e a comunidade tenham condições de se tornarem independentes ${ }^{(10)}$.

O instrumento de VD tem como objetivo promover, manter ou restaurar a saúde, procurando maximizar a independência e minimizar o efeito da doença ou da incapacidade sobre o indivíduo. Procura desenvolver as capacidades da pessoa, a reaprendizagem de novas habilidades e, consequentemente, proporcionar maior autonomia na realização de AVD. Desse modo, contribui para o incremento das premissas da promoção de saúde definidas pela Organização Mundial de Saúde, promovendo e maximizando ganhos em saúde na sua prática ${ }^{(10,12)}$.

Depois da ATQ, o individuo apresenta ainda um conjunto de limitações em suas atividades de vida, que lhe são "impostas" para o sucesso da artroplastia e, consequentemente, para a sua plena recuperação. As medidas de recuperação não se devem limitar a uma intervenção dirigida apenas ao tratamento curativo relacionado a uma situação aguda, mas devem também ser direcionadas às necessidades de apoio nas AVD e aos cuidados de reabilitação.

A VD, como atividade de enfermagem, pode ser vista como um continuum dos cuidados de 
saúde dispensados ao indivíduo ou à família, proporcionados em contexto domiciliário, tendo como objetivo promover, manter ou restaurar a saúde, procurando maximizar a independência e minimizar os efeitos da doença ou da incapacidade do indivíduo. O propósito é facilitar um regresso mais precoce ao domicílio, reduzindo o tempo de internação, diminuindo igualmente o risco de comorbidades, como é o caso da infecção relacionada com os cuidados de saúde. A VD de enfermagem permite a realização de atividades programadas no domicílio, facilitando a esses profissionais conhecerem o contexto social e identificarem as necessidades de saúde das famílias ${ }^{(9)}$.

O programa de VD do enfermeiro comunitário, nesse âmbito, deve incluir ações como ensinar, instruir, treinar, assistir, supervisionar sobre temas como: caminhar com auxílio de um andador ou muletas canadenses; posicionar-se; sentar-se; autocuidado relativo a higiene; vestir-se ou despir-se; uso do sanitário; deitar-se e levantar-se; e informar sobre a existência de ajudas técnicas $^{(13)}$.

Assim, "[...] frente à relevância do cuidado prestado ao paciente submetido a artroplastia do quadril, deve-se incorporar a prática da visita domiciliar como ferramenta para a promoção da saúde" ${ }^{\text {(14:75) }}$. Nessa visita, o enfermeiro pode adotar ações que promovam autonomia e melhora da qualidade de vida relacionada com as AVD. É no âmbito dessa problemática que se insere este estudo, que tem como objetivo avaliar os efeitos da implantação de um programa de visitas domiciliares a pessoas adultas no domínio AVD.

\section{Método}

Estudo de intervenção, do tipo quasi-experimental ${ }^{(15)}$, realizado no setor de ortopedia de um hospital e em contexto comunitário, de um município de Vila Real, localizado ao Norte de Portugal, cuja população total era de $51 \mathrm{mil}$ pessoas em 2011, das quais aproximadamente 18,0\% tinham 65 anos ou mais de idade ${ }^{(16)}$.

A população em estudo constou de 50 usuários internados no Serviço de Ortopedia de um
Centro Hospitalar do Interior Norte de Portugal, no período de julho a dezembro de 2012, com indicação de ATQ, para melhora das condições decorrentes de coxartrose. Destes, 88\% ( $\mathrm{N}=44)$ aceitaram participar do estudo. Os casos $(\mathrm{N}=21)$ foram selecionados no pré-operatório de ATQ e incluídos no Programa de VD do Enfermeiro (intervenção) e compõem o grupo experimental (GE). Os controles ( $\mathrm{N}=23)$ foram os usuários internados pelo mesmo motivo, mas não receberam VD do enfermeiro para implantação do programa, sendo este o grupo de controle (GC). Este grupo seguiu recomendações pós-alta instituídas pelo Centro Hospitalar.

A seleção dos usuários de cada grupo não foi aleatória, tendo sido realizada em função da área de abrangência dos Centros de Saúde. Os usuários que pertenciam à área dos centros de saúde que se disponibilizaram de forma voluntária a seguir o programa de VD foram incluídos no GE e os outros usuários no GC.

O instrumento de coleta de dados foi um formulário constituído por três partes: a primeira, com a caracterização sociodemográfica; a segunda, composta pela escala de Hip Disability and Osteoarthritis Outcome Score (HOOS LK 2.0), validada para a população portuguesa ${ }^{(17)}$; e a terceira, constituída por questões relativas ao programa de VD após a alta hospitalar. A escala HOOS avalia a incapacidade gerada por problemas no quadril, incluindo as AVD. O presente estudo reporta-se apenas ao domínio AVD. Para caracterização socioeconômica, foram coletadas informações sobre sexo, idade, situação conjugal, escolaridade, local de residência (urbana e rural) e condição perante o trabalho (ativo e inativo). A escala foi aplicada em dois momentos de coleta de dados, pré e pós-intervenção (Observação 1 e Observação 2).

O primeiro momento de avaliação foi efetuado no pré-operatório, com o usuário internado; o segundo, foi realizado em contexto comunitário no $3^{\circ}$ mês pós-operatório. A implementação do programa de VD foi efetuada pela equipe de Enfermagem dos Centros de Saúde que abrangiam a área de residência do usuário, instruída previamente para o efeito, a fim de 
uniformizar os procedimentos. A concretização dessas visitas, durante aqueles períodos temporais, prendeu-se ao fato de ser nessa altura que se dava o processo de transição do hospital para o domicílio, onde se tornava necessária a readaptação e a autonomia desses usuários continuava limitada.

O programa de VD contemplou duas visitas agendadas previamente com o usuário. O papel do enfermeiro foi, por meio da ES, oferecer conhecimentos, instruir e treinar os usuários nas suas AVD. A primeira visita permitiu identificar as dificuldades no processo adaptativo ao domicílio e na realização das AVD, tendo sido realizada ES com o usuário, que foi instruído e treinado quanto à marcha, sentar/levantar, deitar/levantar, vestir/calçar, higiene e utilização do sanitário e vigilância das complicações pós-operatórias. Na segunda visita, foi solicitado ao usuário para realizar AVD e treinar aquelas que ainda não tinha realizado, sendo feita a correção de erros e de movimentos que poderiam colocar em risco a integridade da prótese e, novamente, a vigilância de complicações pós-operatórias. Ambos os grupos foram acompanhados na consulta hospitalar e receberam cuidados de rotina dos profissionais dos Centros de Saúde.

Os escores que representaram o domínio AVD foram analisados por meio de estatísticas descritivas (média, mínimo e máximo) e as diferenças quanto às caraterísticas sociodemográficas entre GE e GC foram observadas por meio de testes $\chi^{2}$. A relação entre as médias do domínio AVD obtidas nos dois momentos (O1 e O2), foi verificada em relação a cada uma das variáveis sociodemográficas, por meio de testes não paramétricos (Mann-Whitney e Kruskal-Wallis).

Em seguida, procedeu-se ao pedido de uma MANOVA a dois fatores fixos, para avaliar o efeito do programa de intervenção de VD sobre o domínio AVD. Verificou-se, ao mesmo tempo, o efeito do programa e das variáveis sociodemográficas, no compósito da média da pontuação do domínio AVD, nas duas observações (O1 e O2). Foram colocados sucessivamente como fatores, o programa de VD (GE), com outro fator (variável independente), entre os quais o sexo, faixa etária, situação conjugal, escolaridade e condição perante o trabalho ${ }^{(18)}$. A análise dos dados foi realizada com apoio do software SPSS 20.0 ( (RIBM, 2013).

Para a realização deste estudo, foi solicitada autorização ao Conselho de Administração do Centro Hospitalar, cuja Comissão de Ética emitiu parecer favorável (Protocolo de 27/6/2012). Os procedimentos respeitaram o constante na Declaração de Helsinki.

\section{Resultados}

Do total de participantes $(n=44)$, a maioria era do sexo masculino (61,4\%), pertencia à faixa etária dos 65 e mais anos (65,9\%), com o $1^{\circ}$ e $2^{\circ}$ ciclos de escolaridade $(61,4 \%)$, possuía a situação conjugal de casado $(70,5 \%)$, predominantemente residia em área rural $(77,3 \%)$ e não exercia atividade laboral (79,5\%). Tinham em média 68,3 anos de idade (mínimo 47 e máximo 85 anos). Quanto às características sociodemográficas, não foram verificadas diferenças significativas entre os participantes do grupo de intervenção (GE) e do grupo controle (GC).

A média da pontuação do domínio AVD nos dois grupos em comparação, aumentou da O1 para a $\mathrm{O} 2$, mas o aumento foi mais pronunciado no GE $(77,35)$ que no GC $(70,01)$ da O1 para a O2. Isto significa que a incapacidade relacionada com o autocuidado nas AVD diminuiu da O1 para a O2 (Tabela 1). 
Tabela 1 - Distribuição das médias, mínimo e máximo do domínio Atividades de Vida Diária do Hip Disability and Osteoarthritis Outcome Score por grupo e por observação. Vila Real, Portugal - 2012 (N=44)

\begin{tabular}{lc|c|c|c|c|c|c}
\hline \multirow{2}{*}{ HOOS } & \multicolumn{3}{|c|}{ Grupo controle } & \multicolumn{3}{c}{ Grupo experimental } \\
\cline { 3 - 7 } & & Média & Mínimo & Máximo & Média & Mínimo & Máximo \\
\hline $\begin{array}{l}\text { Domínio } \\
\text { Atividades de }\end{array}$ & $\begin{array}{c}\text { Observação } \\
1\end{array}$ & 25,80 & 2,94 & 48,44 & 31,30 & 11,76 & 62,50 \\
Vida Diária & $\begin{array}{c}\text { Observação } \\
2\end{array}$ & 70,01 & 5,88 & 98,53 & 77,35 & 45,59 & 95,59 \\
\hline
\end{tabular}

Fonte: Elaboração própria.

No cruzamento do domínio AVD com as variáveis de atributo, não se observaram, na O1, diferenças estatísticas significativas; na O2, verificou-se o mesmo, exceto quanto à variável sexo
( $p=0,033)$. Neste caso, o sexo masculino obteve a média de posição mais elevada $(25,78$ versus 17,29 do sexo feminino), isto é, apresentava menor incapacidade nas AVD (Tabela 2).

Tabela 2 - Cruzamento do Domínio Atividades de Vida Diária do Hip Disability and Osteoarthritis Outcome Score com as variáveis sociodemográficas, por observação. Vila Real, Portugal - 2012 (N=44) (continua)

\begin{tabular}{|c|c|c|c|c|c|}
\hline Variáveis & $\mathbf{n}$ & $\begin{array}{c}\text { Média de } \\
\text { posição }\end{array}$ & Valor teste & $\begin{array}{l}\text { Graus de } \\
\text { liberdade }\end{array}$ & Probabilidade \\
\hline $\begin{array}{l}\text { AVD Observação } 1 \text { X Sexo } \\
\text { Masculino } \\
\text { Feminino }\end{array}$ & $\begin{array}{l}27 \\
17\end{array}$ & $\begin{array}{l}15,00 \\
18,53\end{array}$ & MW $=162$ & - & 0,103 \\
\hline $\begin{array}{l}\text { AVD Observação } 2 \text { X Sexo } \\
\text { Masculino } \\
\text { Feminino }\end{array}$ & $\begin{array}{l}27 \\
17\end{array}$ & $\begin{array}{l}25,78 \\
17,29\end{array}$ & $\mathrm{MW}=141$ & - & $\mathbf{0 , 0 3 3}$ \\
\hline $\begin{array}{l}\text { AVD Observação } 1 \text { X Faixa etária } \\
\quad 45-64 \text { anos } \\
\geq 65 \text { anos }\end{array}$ & $\begin{array}{l}15 \\
29\end{array}$ & $\begin{array}{l}24,33 \\
21,55\end{array}$ & $\mathrm{MW}=190$ & - & 0,496 \\
\hline $\begin{array}{l}\text { AVD Observação } 2 \text { X Faixa etária } \\
\quad 45-64 \text { anos } \\
\geq 65 \text { anos }\end{array}$ & $\begin{array}{l}14 \\
30\end{array}$ & $\begin{array}{l}19,89 \\
23,72\end{array}$ & $\mathrm{MW}=173,50$ & - & 0,357 \\
\hline $\begin{array}{l}\text { AVD Observação } 1 \text { X Situação } \\
\text { conjugal } \\
\text { Solteiro } \\
\text { Casado } \\
\text { Viúvo }\end{array}$ & $\begin{array}{c}4 \\
31 \\
9\end{array}$ & $\begin{array}{l}17,75 \\
22,19 \\
25,67\end{array}$ & $\mathrm{KW}=1,113$ & 2 & 0,573 \\
\hline $\begin{array}{l}\text { AVD Observação } 2 \text { X Situação } \\
\text { conjugal } \\
\text { Solteiro } \\
\text { Casado } \\
\text { Viúvo }\end{array}$ & $\begin{array}{c}4 \\
31 \\
9\end{array}$ & $\begin{array}{l}27,13 \\
21,60 \\
23,56\end{array}$ & $\mathrm{KW}=0,733$ & 2 & 0,693 \\
\hline $\begin{array}{l}\text { AVD Observação } 1 \text { X Escolaridade } \\
\text { Não sabe ler e escrever } \\
\text { Sabe ler e escrever } \\
1^{\circ} \text { e } 2^{\circ} \text { ciclos }\end{array}$ & $\begin{array}{c}7 \\
10 \\
27\end{array}$ & $\begin{array}{l}15,00 \\
27,85 \\
22,46\end{array}$ & $\mathrm{KW}=4,127$ & 2 & 0,127 \\
\hline $\begin{array}{l}\text { AVD Observação } 2 \text { X Escolaridade } \\
\text { Não sabe ler e escrever } \\
\text { Sabe ler e escrever } \\
1^{\circ} \text { e } 2^{\circ} \text { ciclos }\end{array}$ & $\begin{array}{c}7 \\
10 \\
27\end{array}$ & $\begin{array}{l}20,21 \\
23,80 \\
22,61\end{array}$ & $\mathrm{KW}=0,326$ & 2 & 0,849 \\
\hline
\end{tabular}


Tabela 2 - Cruzamento do Domínio Atividades de Vida Diária do Hip Disability and Osteoarthritis Outcome Score com as variáveis sociodemográficas, por observação. Vila Real, Portugal - 2012 (N=44) (conclusão)

\begin{tabular}{|c|c|c|c|c|c|}
\hline Variáveis & $\mathbf{n}$ & $\begin{array}{l}\text { Média de } \\
\text { posição }\end{array}$ & Valor teste & $\begin{array}{l}\text { Graus de } \\
\text { liberdade }\end{array}$ & Probabilidade \\
\hline \multicolumn{6}{|c|}{ AVD Observação 1 X Residência } \\
\hline Urbano & 10 & 17,70 & \multirow[t]{2}{*}{$\mathrm{MW}=122$} & \multirow{2}{*}{ - } & \multirow[t]{2}{*}{0,179} \\
\hline Rural & 34 & 23,91 & & & \\
\hline \multicolumn{6}{|c|}{ AVD Observação 2 X Residência } \\
\hline Urbano & 10 & 25,95 & \multirow{2}{*}{$\mathrm{MW}=135,50$} & \multirow[t]{2}{*}{-} & \multirow{2}{*}{0,334} \\
\hline Rural & 34 & 21,49 & & & \\
\hline \multirow{2}{*}{\multicolumn{3}{|c|}{$\begin{array}{l}\text { AVD Observação } 1 \text { X Condição } \\
\text { perante o trabalho }\end{array}$}} & \multirow{4}{*}{$\mathrm{MW}=140$} & \multirow{4}{*}{-} & \multirow{4}{*}{0,610} \\
\hline & & & & & \\
\hline População ativa & 9 & 24,44 & & & \\
\hline População não ativa & 35 & 22,00 & & & \\
\hline \multirow{2}{*}{\multicolumn{3}{|c|}{$\begin{array}{l}\text { AVD Observação } 2 \text { X Condição } \\
\text { perante o trabalho }\end{array}$}} & \multirow{4}{*}{$\mathrm{MW}=126,50$} & \multirow{4}{*}{-} & \multirow{4}{*}{0,367} \\
\hline & & & & & \\
\hline População ativa & 9 & 19,06 & & & \\
\hline População não ativa & 35 & 23,89 & & & \\
\hline
\end{tabular}

Fonte: Elaboração própria.

Legenda: KW: Kruskal-Wallis; MW: Mann-Whitiney; AVD: Atividades de Vida Diária.

Não se observaram diferenças estatísticas significativas ( $p>0,05)$ na análise multivariada do teste MANOVA, a dois fatores fixos, entre o compósito da média da pontuação do domínio AVD, nas duas observações (O1 e O2), o programa de VD (GE) e as variáveis sociodemográficas, exceto, quanto à situação conjugal.

No cruzamento entre o domínio AVD e os fatores programa de VD e a situação conjugal, verificou-se um efeito significativo de média dimensão $\left(\eta^{2} \mathrm{p}=0,126\right)$, na interação do programa de VD com a variável Situação conjugal sobre o compósito multivariado (AVD O1 e AVD O2) (Traço de Pilai=0,251; F de Snecdor [2,39]=2,732; $p=0,035$; Potência=0,729). A potência apurada é, no entanto, média. Isto quer dizer que a interação das variáveis programa de VD e situação conjugal teve efeito no compósito do domínio AVD na O1 e O2 da HOOS, sendo, sobretudo, os idosos solteiros os que se beneficiaram mais com o programa, apresentando menor incapacidade funcional nas AVD, na observação O2, comparando com a observação O1 (médias na O2 dos idosos viúvos, solteiros e casados, respectivamente, 84,04; 81,74 e 73,76 versus O1, respectivamente, 42,46; 21,63 e 29,24). Nos idosos viúvos e casados, essa melhora foi menos pronunciada.

A MANOVA relativa ao fator programa de VD revelou não existir um efeito significativo, na interação do programa de VD sobre o compósito multivariado (AVD O1 e AVD O2) (Traço de Pilai $=0,084 ; Z[1,42]=1,888 ; \mathrm{p}=0,164$; Potência $=0,370)$. No entanto, a média neste domínio aumenta da O1 para a O2, sendo sempre mais elevada no GE do que no GC. Isto significa que o programa de VD teve algum efeito na melhora da média do domínio (a média do GE na O2 foi de 77,35, enquanto a do GC foi de 70,01), mas sem diferenças estatísticas significativas.

\section{Discussão}

Os usuários participantes do estudo eram majoritariamente do sexo masculino $(60,09 \%)$, em ambos os grupos, o que difere da maior parte da bibliografia consultada, que diz que a OA é mais prevalente no sexo feminino. No entanto, esses resultados estão de acordo com os obtidos no estudo realizado em Coimbra (Portugal) ${ }^{(19)}$, no 
qual $62,5 \%$ de sujeitos era do sexo masculino, e também com o estudo realizado em Minas Gerais (Brasil) ${ }^{(20)}$, que encontrou 55,3\% de indivíduos do sexo masculino. A média de idades era 68,3 anos, o que corrobora a bibliografia, que refere a predominância da coxartrose na segunda metade de vida e a prevalência das alterações degenerativas artrósicas entre 55 e 65 anos e mais de idade ${ }^{(21)}$.

Quanto às habilitações acadêmicas, constatou-se que $61,4 \%$ tinha o $1^{\circ}$ e $2^{\circ}$ ciclos, mas $15,9 \%$ não sabiam ler nem escrever, o que está de acordo com o último censo da população portuguesa ${ }^{(22)}$, em que, na zona Norte do país, 15,62\% dos indivíduos não possuíam nível de escolaridade completo e $54,89 \%$ possuíam o $1^{\circ}$ ou $2^{\circ}$ ciclos de escolaridade. Este resultado está ainda em consonância com outro estudo realizado em Lisboa ${ }^{(21)}$, no qual os sujeitos participantes possuíam, como habilitações literárias, o ensino básico ou inferior, em que $63,3 \%$ sabiam ler e escrever e 22,2\% possuíam o $1^{\circ}$ ciclo, isto é, tratava-se de uma amostra com baixa escolaridade, tal como a deste estudo.

No que diz respeito à situação conjugal, os indivíduos de ambos os grupos eram majoritariamente casados (70,05\%), o que vai ao encontro de estudos realizados em Coimbra e Lisboa ${ }^{(19,21)}$, cujos sujeitos, em sua maioria, eram casados, tendo obtido as percentagens de $87,5 \%$ e $64,0 \%$, respectivamente, resultados que podem estar relacionados com a faixa etária dos participantes do estudo.

Neste estudo, apesar de não se terem verificado diferenças estatísticas, a média do domínio AVD da escala HOOS aumentou sempre da O1 para a O2, quer no GC, quer no GE. Entretanto, essas diferenças foram mais substanciais no último grupo. O mesmo sucedeu no estudo realizado na Itália ${ }^{(23)}(n=145)$, com pretensão de traduzir e validar a escala HOOS para a população italiana, que verificou a existência de um aumento da média da pontuação em todos os domínios, da O1 para a O2, após ATQ.

A média da pontuação do domínio AVD na O2 diferiu entre os sexos, sendo superior no sexo masculino. Esta diferença entre os dois sexos pode ser explicada pelo fato de o homem ter melhor autopercepção do estado de saúde, como refere o autor de outro estudo ${ }^{(24)}$ realizado em Portugal $(n=300)$, com o objetivo de avaliar a QV relacionada à saúde de pessoas idosas segundo o sexo. Outro estudo realizado em Bragança, Portugal $^{(25)}$, com o objetivo de avaliar a percepção da Qualidade de Vida Relacionada à Saúde (n=1111 usuários, maiores de 18 anos, inscritos nos Centros de Saúde do ACeS Trás-os-Montes I - Nordeste), verificou que os valores médios da QV relacionados com as AVD eram maiores no sexo masculino, tal como no presente estudo.

A análise multivariada efetuada possibilitou a apuração de um efeito estatístico significativo no cruzamento entre o conjunto das médias AVD O1 e AVD O2, com o Programa de VD e a variável situação conjugal, sendo este resultado relativo à interação situação conjugal com o Programa de VD. É possível inferir-se que esse resultado pode ser atribuído ao programa de VD, que foi mais útil aos usuários que não tinham companheiro(a).

O aumento generalizado da média do domínio AVD do HOOS, em ambos os grupos, pode explicar-se pela realização da ATQ, a que todos os sujeitos participantes foram submetidos. Por sua vez, o aumento mais substancial verificado no GE pode dever-se à aplicação do programa de VD, a que este grupo foi sujeito ${ }^{(21)}$. No entanto, a ausência de uma diferença estatística significativa entre a média dos dois grupos em análise pode dever-se ao fato de terem sido realizadas apenas duas VD, no âmbito da intervenção no GE, que não terá sido suficiente para um aumento significativo da média desse grupo.

Reconhecem-se algumas limitações a este estudo, sobretudo no que se relaciona com a utilização de uma amostra não-probabilística, de conveniência, o que limita a generalização dos resultados para esta população.

\section{Conclusão}

Pode-se afirmar que as variáveis sociodemográficas não influenciaram o domínio AVD da escala, entre os participantes do estudo, e que 
o programa de VD provocou um aumento na média desse domínio, sobretudo no GE, que não foi, no entanto, estatisticamente significativo. Conclui-se que o referido programa não teve efeito nas AVD. A variável sexo influenciou o domínio AVD na O2, tendo os homens percebido menor incapacidade na realização das AVD.

A interação do programa de intervenção de VD de Enfermagem com a situação conjugal influenciou o conjunto AVD O1-AVD O2, sendo esse efeito visível apenas na O2, tendo-se verificado que os usuários solteiros, que provavelmente vivem sozinhos, parecem beneficiar-se mais do referido programa. A incapacidade relacionada com as AVD melhorou da O1 para a O2, embora sem diferenças estatísticas significativas, o que poderá ser resultado da implementação do programa de intervenção de VD de enfermagem, no desempenho das AVD dos utentes do GE.

O estudo realizado já teve implicações nos cuidados de enfermagem prestados, com a implementação do programa de VD. Os enfermeiros que colaboraram nessa implementação estão agora mais sensibilizados para a importância da VD e do apoio que podem dar a esse grupo, de uma situação de doença para uma situação de mais saúde, no processo de transição de uma unidade hospitalar para a comunidade. A sua continuidade será uma mais-valia para a qualidade de vida e autocuidados e obtenção de ganhos em saúde.

A realização de novos estudos na área poderá revelar-se importante para a relação terapêutica entre a enfermagem comunitária e o indivíduo submetido a ATQ, podendo, este estudo, servir como ponto de partida para investigações futuras, mais prolongadas no tempo, com maior amostragem e aleatoriedade, que permitam corroborar ou não as presentes conclusões.

\section{Colaborações:}

1 - concepção, projeto, análise e interpretação dos dados: António Manuel Rocha Fonseca Pinto, Elisabete Pimenta Araújo Paz e Amâncio António de Sousa Carvalho;
2 - redação do artigo e revisão crítica relevante do conteúdo intelectual: António Manuel Rocha Fonseca Pinto, Elisabete Pimenta Araújo Paz e Amâncio António de Sousa Carvalho;

3 - aprovação final da versão a ser publicada: António Manuel Rocha Fonseca Pinto, Elisabete Pimenta Araújo Paz e Amâncio António de Sousa Carvalho.

\section{Referências}

1. World Health Organization. Global strategy and action on ageing and health. Geneva; 2017 [cited 2019 Aug 23]. Available from: https://www.who. int/ageing/WHO-GSAP-2017.pdf?ua=1

2. Tavares RE, Jesus MCP, Machado DR, Braga VAS, Tocantins FR, Merighi MAB. Envelhecimento saudável na perspectiva dos idosos: uma revisão integrativa. Rev Bras Geriatr Gerontol. 2017;20(6):889-900. DOI: $10.1590 / 1981-22562017020.170091$

3. Cunha-Miranda L, Faustino A, Alves C, Vicente V, Barbosa S. Avaliação da magnitude da desvantagem da osteoartrite na vida das pessoas: estudo MOVES. Rev Bras Reumatol [Internet]. 2015 [cited 2017 Mar 14];55(1):22-30. Available from: http://www.scielo. br/pdf/rbr/v55n1/0482-5004-rbr-55-01-0022.pdf

4. Oliveira NC, Alfieri FM. Exercícios resistidos na osteoartrite: uma revisão. Acta Fisiatr [Internet]. 2014 [cited 2019 Aug 22];21(3):141-6. Available from: http://www.actafisiatrica.org.br/ detalhe_artigo.asp?id=555

5. Ferreira L, Gomes E. Papel da glucosamina no alívio dos sintomas da osteoartrose: revisão baseada na evidência. Rev Port Med Geral Fam [Internet]. 2014 [cited 2019 Aug 22];30:370-84. Available from: http://www.scielo.mec.pt/pdf/ rpmgf/v30n6/v30n6a06.pdf

6. Liga Portuguesa Contra as Doenças Reumáticas. Início. LPCDR. Lisboa; 2019 [cited 2019 Aug 22]. Available from: https://www.lpcdr.org.pt/

7. Faustino A. Epidemiologia e importância económica e social das doenças reumáticas: estudos nacionais. Acta Reumatol Port. 2002;27:21-36.

8. Pinto AM, Carvalho A. Efeito de um programa de visitação domiciliária de enfermagem na qualidade de vida dos doentes com coxartrose submetidos a artroplastia total da anca. In: Abreu M, Ribeiro TT, Teixeira M, Peixoto MJ, Araújo F, Freire RM, 
et al. organizadores. Jornadas Internacionais de Enfermagem Comunitária 2014. Livro de comunicações \& conferências. Porto: Escola Superior de Enfermagem do Porto; 2016. p. 116-24.

9. Andrade AM, Guimarães AMDN, Costa DM, Machado LC, Gois CFL. Visita domiciliar: validação de um instrumento para registro e acompanhamento dos indivíduos e das famílias. Epidemiol Serv Saúde. 2014 jan-mar;23(1):165-75. DOI: $10.5123 /$ S1679-49742014000100016

10. Couto AM, Hell CAI, Lemos IF, Castro EAB. Cuidado domiciliar sob a ótica de idosos dependentes: contribuições para a Enfermagem. Rev baiana enferm. 2016 out-dez;30(4):1-12. DOI: 10.18471/ rbe.v30i4.16068

11. Arnemann CT, Lavich CRP, Terra MG, Mello AL, Raddatz M. Educação em saúde e educação permanente: ações que integram o processo educativo da Enfermagem. Rev baiana enferm. 2018;32:e24719. DOI: 10.18471/rbe.v32.24719

12. Rocha KB, ConzJ, Barcinsky M, Paiva D, Pizzinato A. A visita domiciliar no contexto da saúde: uma revisão da literatura. Psicol Saúde Doenças. 2017;18(1):170-85. DOI: 10.15309/17psd180115

13. Centro Hospitalar de Lisboa Norte. Manual informativo para o doente com osteoartrose [Internet]. Lisboa; 2019 [cited 2019 Aug 22]. Available from: http://www.chln.pt/media/k2/ attachments/servico_reumatologia/Manual $\% 20$ da\%20Osteoartrose.pdf

14. Oliveira MLP, Jansen MM, Almeida MA. Visita domiciliar pré-operatória no programa de cuidado de enfermagem em artroplastia total de quadril. Rev HCPA. 2007;27(2):74-6.

15. Vilelas J. Investigação. O processo de construção do conhecimento. 2a ed. Lisboa, PT: Edições Sílabo; 2017.

16. Instituto Nacional de Estatística. Estimativas anuais de População Residente, Portugal, NUTS II, NUTS III e municípios - exercício Adhoc 2010 e 2011. Lisboa, PT; 2012.

17. Nunes S, Cabri J, Gil J. Measuring health-related quality of life in patients with hip osteoarthritis and total hip replacement: Adaption and validation of the hip disability and osteoarthritis outcome source LK 2.0 (HOOS 2.0) to the portuguese culture [Internet]. Coimbra; 2009 [cited 2012 Jun 2]. Available from: http://www.koos.nu/ hoosportuguese.pdf

18. Marôco J. Análise estatística com o SPSS Statistics. Pêro Pinheiro, PT: Report Number; 2014.

19. Reis PO. Fatores que influenciam a capacidade funcional em indivíduos submetidos a Artroplastia Total da Anca [dissertação]. Coimbra, PT: Escola Superior de Enfermagem de Coimbra; 2016.

20. Loures EA, Leite ICG. Análise da qualidade de vida de pacientes osteoartrósicos submetidos à artroplastia total do quadril. Rev Bras Ortop [Internet]. 2012 [cited 2016 Jun 9];47(4):498-504. Available from: http://www.scielo.br/scielo.php?script=sci_ arttext\&pid=S0102-36162012000400017

21. Nunes SCF. Medição da qualidade de vida relacionada com a saúde em doentes com osteoartrose da anca e artroplastia da anca: adaptação e validação da hip disability and osteoarthritis outcome score lk 2.0 (hoos 2.0) para a cultura portuguesa [dissertação]. Lisboa, PT: Faculdade de Motricidade Humana, Universidade Técnica de Lisboa; 2012.

22. Instituto Nacional de Estatística. Censos 2011: Resultados definitivos. Lisboa, PT; 2012.

23. Torre M, Luzi I, Mirabella F, Manso M, Zanoli G, Tucci G, et al. Cross-cultural adaptation and validation of the Italian version of the Hip Disability and Osteoarthritis Outcome Score (HOOS). Health Qual Life Outcomes. 2018;16(1):115. doi: 10.1186/ s12955-018-0935-6

24. Daniel F, Monteiro R, Antunes S, Fernandes R, Ferreira PL. Qualidade de vida relacionada com a saúde de pessoas idosas numa perspetiva de género. Port J Public Health. 2018;36(2):59-65. DOI: $10.1159 / 000490929$

25. Praça MIF. Qualidade de vida relacionada com a saúde: a perspectiva dos usuários que frequentam os Centros de Saúde do ACES Trás-os-Montes I Nordeste [dissertação]. Bragança, PT: Instituto Politécnico de Bragança; 2012.

Recebido: 2 de setembro de 2019 Aprovado: 24 de outubro de 2019 Publicado: 16 de março de 2020 


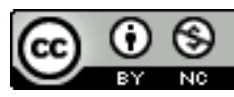

A Revista Baiana de Enfermagem utiliza a Licença Creative Commons - Atribuição-NãoComercial 4.0 Internacional. https://creativecommons.org/licenses/by-nc/4.0/

Este artigo é de acesso aberto distribuído sob os termos da Licença Creative Commons (CC BY-NC).

Esta licença permite que outros remixem, adaptem e criem a partir do seu trabalho para fins não comerciais. Embora os novos trabalhos tenham de lhe atribuir o devido crédito e não possam ser usados para fins comerciais, os usuários não têm de licenciar esses trabalhos derivados sob os mesmos termos. 